\title{
Characterising neovascularisation in fracture healing with laser Doppler and micro-CT scanning
}

\author{
W. Macdonald $\cdot$ S. J. Shefelbine
}

Received: 3 October 2012/ Accepted: 9 July 2013/Published online: 24 July 2013

(C) The Author(s) 2013. This article is published with open access at Springerlink.com

\begin{abstract}
Vascularity of the soft tissues around a bone fracture is critical for successful healing, particularly when the vessels in the medullary canal are ruptured. The objective of this work was to use laser Doppler and microcomputer tomography (micro-CT) scanning to characterise neovascularisation of the soft tissues surrounding the fracture during healing. Thirty-two Sprague-Dawley rats underwent mid-shaft osteotomy of the left femur, stabilised with a custom-designed external fixator. Five animals were killed at each of 2, 4 days, 1, 2, 4 and 6 weeks postoperatively. Femoral blood perfusion in the fractured and intact contralateral limbs was measured using laser Doppler scanning pre- and post-operatively and throughout the healing period. At sacrifice, the common iliac artery was cannulated and infused with silicone contrast agent. MicroCT scans of the femur and adjacent soft tissues revealed vessel characteristics and distribution in relation to the fracture zone. Blood perfusion dropped immediately after surgery and then recovered to greater than the pre-operative level by proliferation of small vessels around the fracture zone. Multi-modal imaging allowed both longitudinal functional and detailed structural analysis of the neovascularisation process.
\end{abstract}

Keywords Fracture healing - X-ray micro-tomography · Angiogenesis - External fixators · Laser Doppler flowmetry

W. Macdonald ( $\square)$

Department of Bioengineering, Imperial College,

South Kensington, London SW7 2AZ, UK

e-mail: w.macdonald@imperial.ac.uk

S. J. Shefelbine

Department of Mechanical and Industrial Engineering,

Northeastern University, Boston, MA 02115, USA

\section{Introduction}

Fracture healing of bone is a complex process affected by systemic, biological and mechanical factors, the combination of which can lead to successful and complete repair of a fracture, or deficiencies of which can cause delayed healing or even non-union.

Angiogenesis is an important early phase of fracture healing, leading to invasion of the initial haematoma by fine vessels and subsequent conversion into soft callus. Impaired angiogenesis can compromise fracture healing [8, 14], whereas enhanced vascularity is known to improve fracture healing [37, 43]. Neovascularisation of the haematoma occurs from the adjacent soft tissues [4, 9, 10, 13], especially when the medullary supply has been disrupted [12].

Neovascularisation in fracture healing has been analysed with a wide variety of techniques. Standard histology uses staining to visualise tissue types or tissue components (such as smooth muscle or endothelial cells) in the fracture callus and surrounding tissue [3, 21, 32, 42]. Immunohistochemistry techniques label proteins particular to blood vessels, such as VEGF and CD31, to identify regions of angiogenesis and quantify small vessels $[22,28,36]$. Capillary proliferation around the fracture site has been demonstrated by micro-angiography [29], and vascular budding adjacent to the fracture defect was revealed with contrast perfused histological sections [23]. Electron microscopy $[21,23,38,46]$ and intra-vital microscopy [47, 49] provide high-resolution images that can indicate fine details of the angiogenesis process, such as changes in cell morphology, gene expression or endothelial cell activity. These techniques can indicate fine details of the vessel structure and distribution, but most are two-dimensional and static, providing little quantitative information on blood flow. 
Detailed three-dimensional structural information is provided by CT scanning with the vessels perfused with contrast resin at killing $[2,34]$, or in vivo [20, 26, 30, 31], but these are often limited snapshots at particular time points and provide no information of the time course of neovascularisation. For example, Tomlinson and associates [40] used silicone contrast perfusion of vessels and microcomputer tomography (micro-CT) scanning of forelimb stress fractures at 3 and 7 days after loading-induced stress fractures. However, scanning in vivo can give contrast and partial filling difficulties [7].

Measures of blood flow or blood perfusion are possible with probe-based laser Doppler [6, 15-17], ultrasound [35], radioactive tracer clearance $[1,33,45,48]$ or functional CT [18-20, 39]. These functional measures must be performed in vivo and provide temporal analysis of the neovascularisation process throughout the fracture healing period. Probe laser Doppler measures at discrete points (sometimes several across a region) and tracer clearance can be used to provide a regional integral of the measured flow. Laser Doppler scanning, however, scans continuously over a region and provides $2 \mathrm{D}$ surveys of blood perfusion, which can be used to identify vessels and regional flow patterns.

In this study, we combined laser Doppler scanning (for longitudinal percutaneous information) and micro-CT imaging (for end-point three-dimensional structural information) to investigate the location and extent of neovascularisation of the soft tissues around the fracture gap during healing.

\section{Methods}

Thirty-two Sprague-Dawley rats (350-500 g; 12-20 weeks of age) underwent left mid-femoral osteotomy fixed with an external fixator. All procedures were approved by the Imperial College, London, ethical review process and were strictly conformed to the Animals (Scientific Procedures) Act 1986 UK Home Office guidelines. Experiments were performed under a Home Office Licence PPL 70/6472 (the conditions of which also fulfil the US NIH guide for the care and use of laboratory animals).

Inhalation anaesthesia was induced in an induction chamber (4\% isoflurane in oxygen at $2 \mathrm{~L} / \mathrm{min}$; IsoFlo, Abbott Laboratories Ltd., Maidenhead, SL6 4XE, UK), the animal was transferred to a rat mask and anaesthesia was maintained with $1 \mathrm{~L} / \mathrm{min}$ oxygen and isoflurane varied as appropriate between 1.5 and $3 \%$ to maintain full anaesthesia and analgesia but to allow optimal recovery. Prophylactic antibiotics (enrofloxacin; Baytril $0.05 \%$, Bayer plc. Newbury, RG14 1JA, UK) and analgesia (buprenorphine; Vetergesic $0.3 \mathrm{mg} / \mathrm{ml}$.; Reckit Benckiser Healthcare (UK) Ltd, Hull, HU8 7DS, UK) were administered in appropriate doses; both thighs were shaved and laser Doppler scanned (model LDI2-HR, Moor Instruments plc, Axminster, England). The animal was then prepared recumbent on its side on a heating mat, surgically draped and skin disinfected for surgery. The left femur was exposed through a lateral skin incision and blunt dissection, four stainless steel fixation pins (1.25 $\mathrm{mm}$ thread diameter) were inserted transversely $7 \mathrm{~mm}$ apart along the femoral length and the femur was cut transversely mid-shaft using a fine hand saw (Fig. 1). The muscle layers and skin were closed (and sutured with resorbable suture material), the pin shafts were passed retrograde through the skin and a custom-designed unilateral fixator was assembled to the pin ends. After rehydration by injection of two $2 \mathrm{ml}$ aliquots of sterile water subcutaneously, the animal was awoken with administration of pure oxygen through the anaesthesia equipment under observation and once fully awake was returned to standard housing. All animals were housed individually with analgesic (rimadil) and antibiotic cover (enrofloxacin $0.05 \%$ ) in the drinking water for 4 days. Thereafter, the animals were housed in threes and allowed water and standard laboratory feed ad libitum.

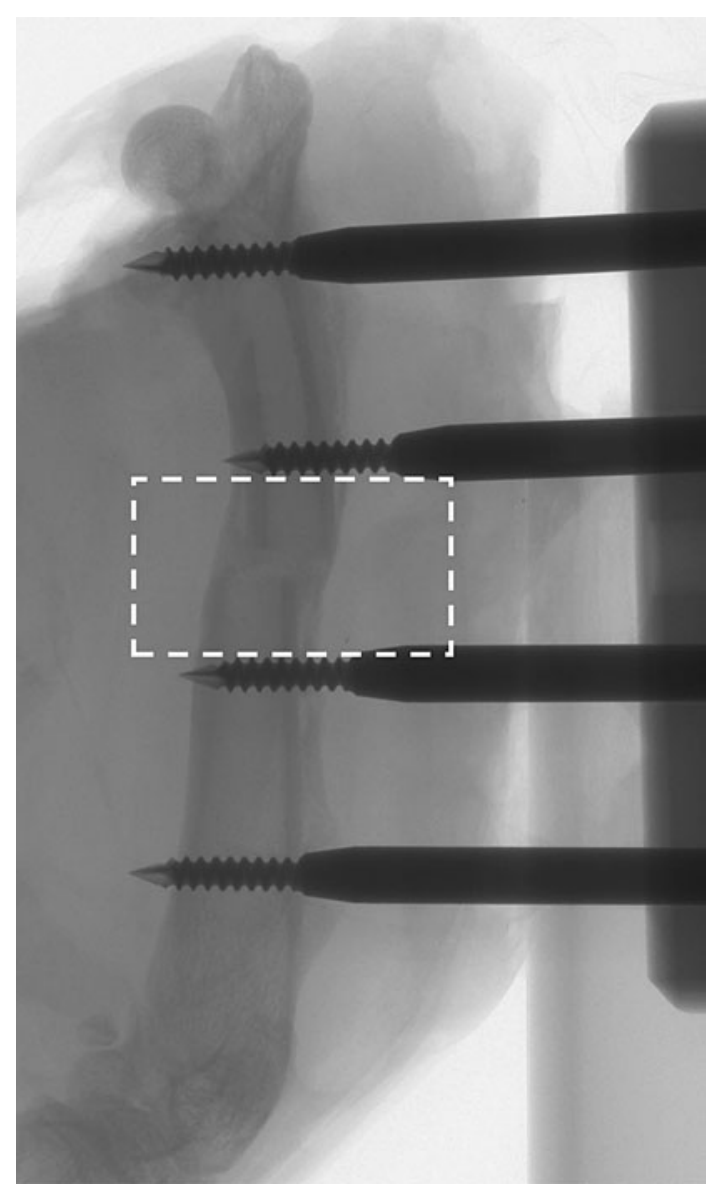

Fig. 1 Radiograph of healed fracture (and fixator) sacrificed 6 weeks after fracture. The fracture zone is outlined 
The full medial aspect of both thighs was laser Doppler scanned $(2.5 \mathrm{~cm} \times 2.5 \mathrm{~cm})$ at a resolution of $0.1 \mathrm{~mm}$, preand post-operatively and at 1,2, 4 and 7 days and thence weekly until sacrifice. Animals were killed at 2 and 4 days post-operatively, and at 1, 2, 4 and 6 weeks post-injury ( $n=5$ per group).

At sacrifice, the anaesthetised animal was laser Doppler scanned, and then the common iliac artery was cannulated, heparinised and infused with silicone contrast agent (Microfil MV-120, Flow Tech Inc., Carver, Mass. USA). The animal was immediately euthanised with pentobarbital, and the hindquarters were harvested and fixed in $10 \%$ neutralbuffered formalin solution for at least 2 weeks.

From laser Doppler scans, the femoral artery was identified by its bifurcation and location, and four regions of interest were analysed: (1) femoral artery, (2) distal femoral artery, (3) the zone cranial to the artery (adjacent to the fracture) and considered to be the tissue most probably involved in neovascularisation of the fracture site $[13,23$, 29] and (4) the zone caudal to the artery (Fig. 2). Proprietary software (Moor LDI V5.1, Moor Instruments plc, Axminster, England) computed mean and maximum perfusion in each region; the mean values were used in subsequent analysis. Daily variations in perfusion due to depth of anaesthesia or body temperature were determined by calculating the change of perfusion in each region in the contralateral leg relative to the pre-operative value in that region (DailyVar). We divided the perfusion values of the fractured leg by this parameter to account for fluctuations not associated with the neovascularisation process. Mean perfusion measures (corrected for daily variations) were then expressed as a percentage change from the pre-operative value for the fractured leg. Inter-operator repeatability of the laser Doppler analysis was determined by three separate investigators analysing 20 separate scans independently. Intra-operator repeatability was determined by one-operator repeating analysis on 20 scans four times.

Micro-CT scanning of the limb-fixator constructs and intact contralateral femora was performed at $180 \mathrm{kV}$ and $133 \mathrm{~mA}$ and a resolution of 21 microns (HMX ST225, $\mathrm{X}$-Tek Systems Ltd. Tring, UK). The excised femora were typically $30 \mathrm{~mm}$ in length and thus consisted of about 1,450 slices. The CT scans of both limbs were reconstructed to show the bone and the vessels (Fig. 3). The steel fixator pins caused some artefactual whiteout of the scans at their level, masking some of the vascular regions on those sections, but the fracture zone (which is the region of interest) was between the middle pins. Scans of fractured limbs were therefore analysed just between the pins on either side of the fracture site (proximally and distally, pins number 2 and 3), a length of about $8 \mathrm{~mm}$. Intact limbs were analysed over the mid-third of the femur, which corresponded to the same level as the fracture region on the contralateral limb. The image stack of the scan was then thresholded automatically ("Trainable Segmentation", Fiji Image Manipulation Software, http://fiji.sc/wiki/index.php/ Fiji) to identify four regions: bone, vessel, tissue and air; the contrast medium-filled vessels displayed CT density midway between bone and soft tissue. The scans were binarised and manipulated to smooth and cohere the vessel regions and minimise all other regions. An automated particle counter (Fiji) was then used to count the vessels on each cross-section, the total cross-sectional (vessel) area and area fraction for each section, and the size and number of vessels in each slice were output to a spreadsheet. These could then be expressed as graphs of vessel number. The
Fig. 2 Laser Doppler scan with four regions of interest identified: 1 femoral artery, 2 distal femoral artery, 3 cranial region overlying the fracture zone and 4 caudal region

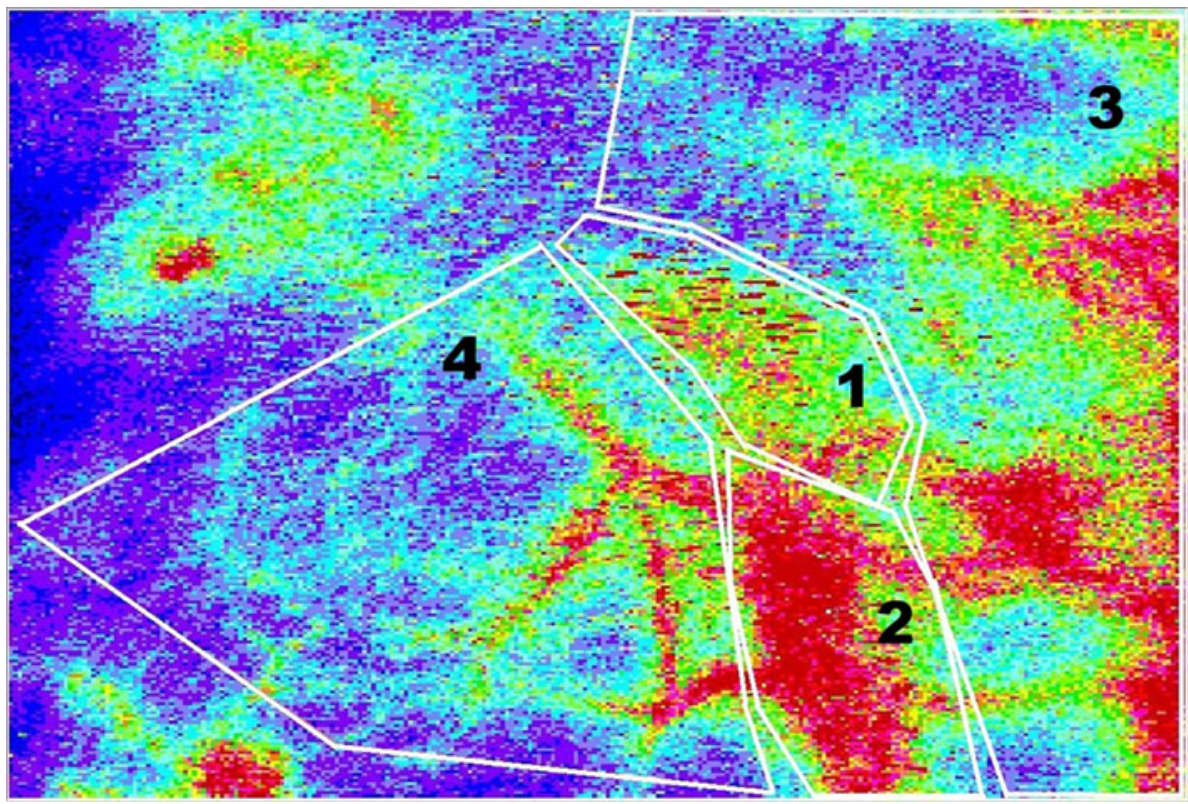


Fig. 3 Three-dimensional reconstruction of $\mu$-CT of bone 6 weeks after fracture (medial view). Pins were left in during scanning to preserve fracture morphology, resulting in artefacts at the level of the pins (gaps in the scan). This did not affect characterisation of vessels in the fracture gap. a Whole bone without vessels (the united fracture site can be seen midshaft, and the pin locations are indicated by dotted lines).

b Whole bone and vessels (the femoral artery is clearly seen running across the medial aspect of the central zone of the femur)
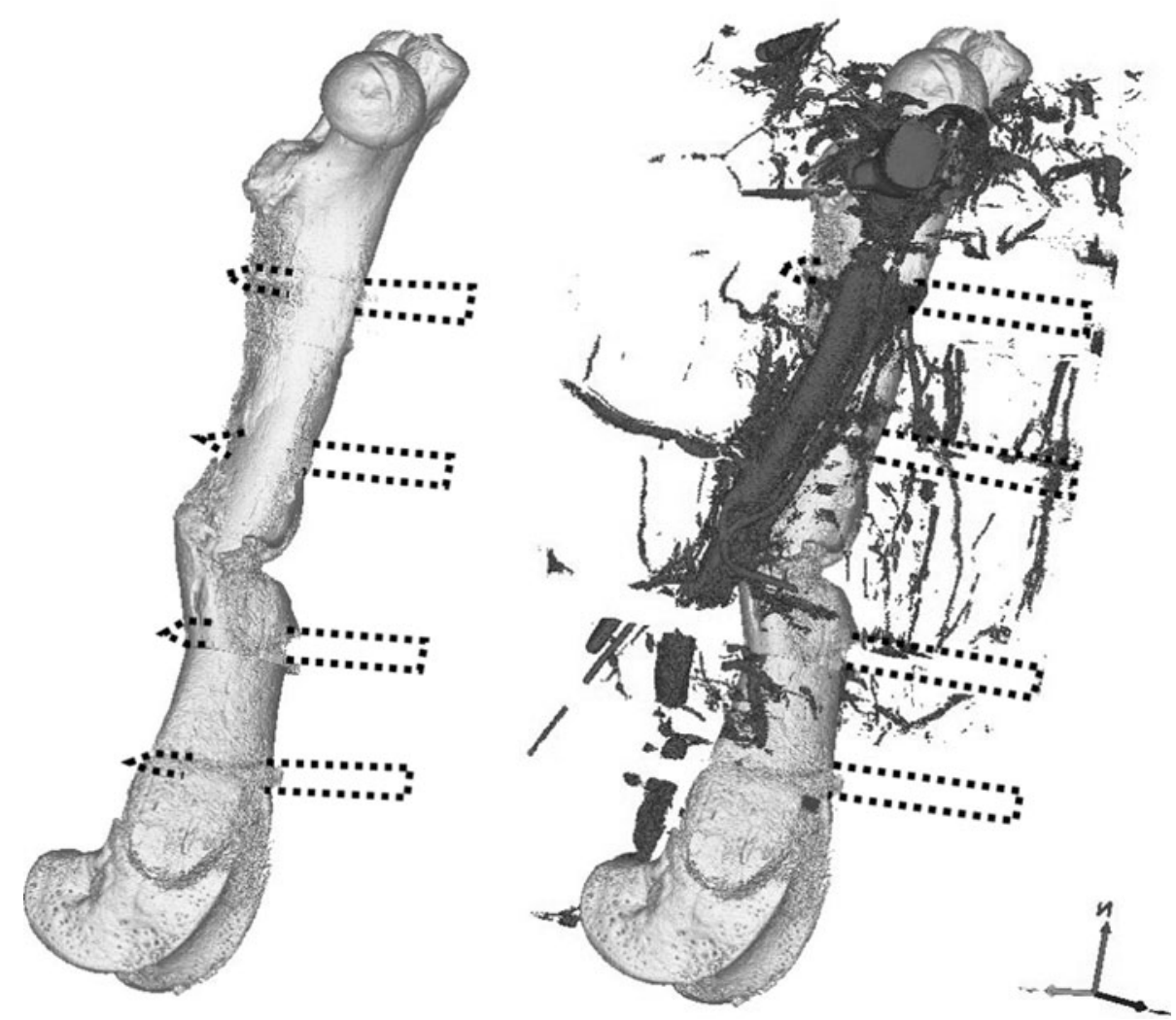

particle counter also reported the size of every particle counted (cross-sectional area of each vessel at each slice) in voxels, which was recorded, and the distribution of sizes was characterised in decades $(1,2,5,10,50,100,500$, 1,000 and 5,000 voxels, corresponding to vessels of crosssectional area of less than 20 , then approximately 30,50 , $70,150,200,500$ and $1,000 \mu \mathrm{m}^{2}$ ). The proportion of small vessels was then calculated as the number of vessels whose cross-section was two voxels or less, summed for all slices in the fracture region divided by the total number of vessels in that region.

Statistical analysis of the perfusion data was performed using one-way analysis of variance (ANOVA) and also a multilevel (growth) model in SPSS (IBM SPSS Statistics, version $20^{\odot}$ 2011, IBM Corp. New York, USA). The growth model was used to analyse the raw perfusion measures from regional analysis considering the effects of time under a linear and quadratic model and using the corresponding measures in the intact leg (Intact), cranial (Cranial) and Cranial Intact and body temperature measures as covariates. Anova was used to test for differences between the fractured side and intact side measures. Statistical analysis of the repeatability studies was undertaken using Spearman's correlation in SPSS.

Vessel density (vessel count) and vessel size in the study (fractured) limb were compared to those in the contralateral (intact) limb for the same region and at the same time point using paired Student's $t$ tests with the Bonferroni correction.

\section{Results}

One cannulation failed, preventing contrast infusion, and two animals' scans were compromised by wound difficulties (although they were still available for analysis). One longer term animal developed respiratory distress and was killed 1 week early (at 5 weeks). Therefore, 31 valid animals were micro-CT analysed at 2 days $(n=4), 4$ days $(n=5), 6$ days (1), 1 week $(n=5), 2$ weeks $(n=6)$, 4 weeks $(n=5), 5$ weeks $(1)$ and 6 weeks $(n=4)$. All 6 -week animals appeared successfully healed on radiographic projection (Fig. 1).

Inter-operator repeatability studies of the laser Doppler analysis gave a Spearman's correlation coefficient of $r=0.88$ for femoral artery regions and 0.83 for the cranial region. Spearman's correlation coefficients for intra-operator repeatability were 0.96 and 0.94 for the femoral artery and cranial region, respectively.

Blood perfusion in the femoral artery (region 1) and the cranial region (region 3, which is adjacent to the fracture region) dropped immediately after operation, increased greatly from 4 days post-operatively to 2 weeks and then declined. The two other regions (2 and 4) showed similar 
trends to a lesser degree (Fig. 4). The mean regional perfusion values over all animals also demonstrated this trend (Fig. 5).

Statistical analysis confirmed these observations; multilevel statistical analysis showed that time after fracture significantly predicted perfusion in the femoral zone of the fractured leg, $F(1,169.8)=4.042, p=0.046$, and the corresponding measures on the intact limb also significantly predicted perfusion $[F(1,181.7)=8.83, p=0.003]$. The dependence on time was significantly better represented by the quadratic model than a linear growth model $\left[\chi^{2}(1) 9.858, p<0.05\right]$, and inclusion of the intact measure was highly significant in improving the model $\left[\chi^{2}(5)\right.$
21.213, $p<0.001]$. Body temperature did not significantly predict perfusion $[F(1,187.92)=0.167, p=0.664]$ nor was there significant interaction between time and the perfusion measures $[F(1,137.6)=1.165, p=0.282]$.

Anova demonstrated a highly significant difference between the femoral (fractured limb) perfusion measure and the intact side $(F=24.5, p<0.001)$, with post hoc comparisons using the least-significant difference (LSD) technique indicating significant differences between preoperative perfusion and post-fracture measures at days 2-14: immediate post-fracture perfusion was highly significantly different $(p<0.001)$ from all time points except 35 days ( $p=0.018$ from pre-fracture perfusion). Perfusion
Fig. 4 Laser Doppler scans at successive time points in a typical subject (above) and the corresponding plot of mean perfusion by zones
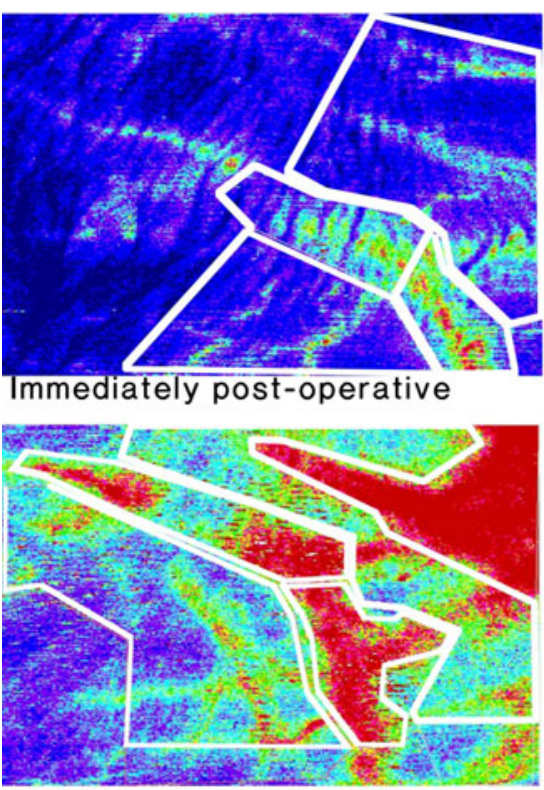

Two days post-operative

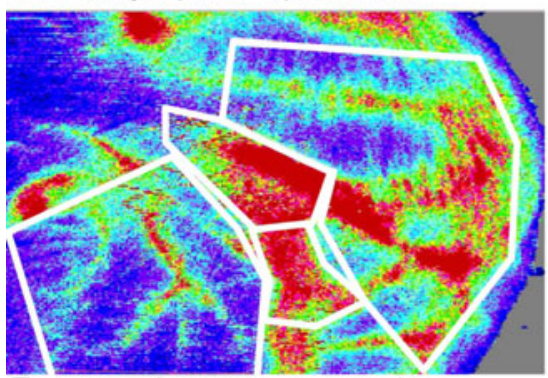

Two weeks post-operative
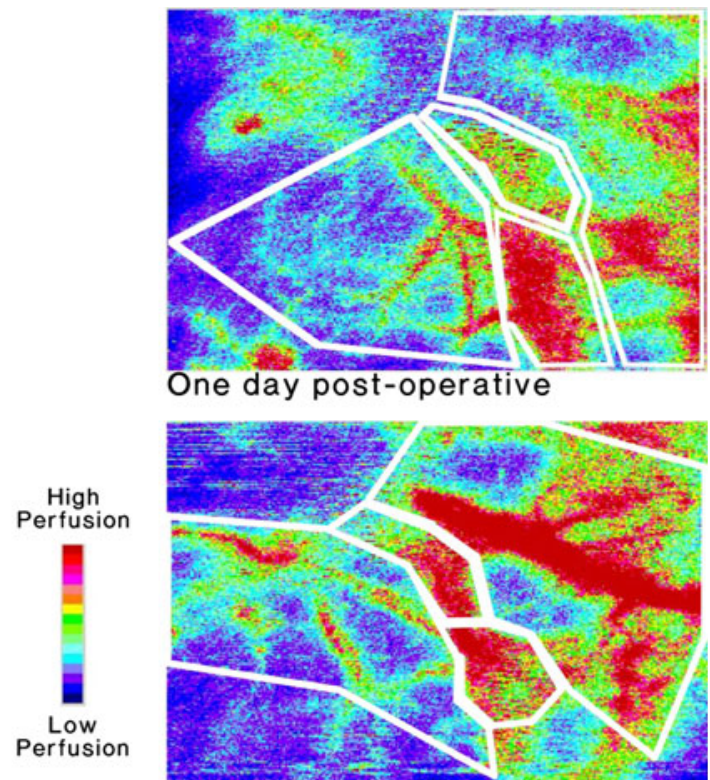

One week post-operative

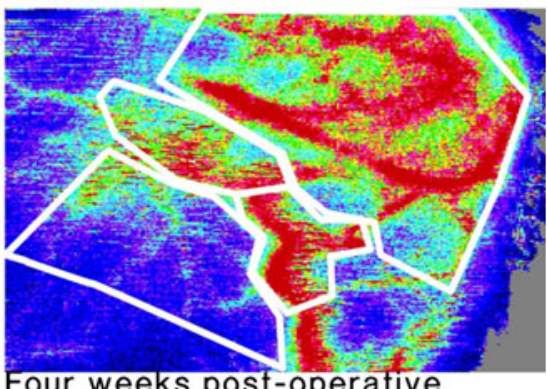

Four weeks post-operative

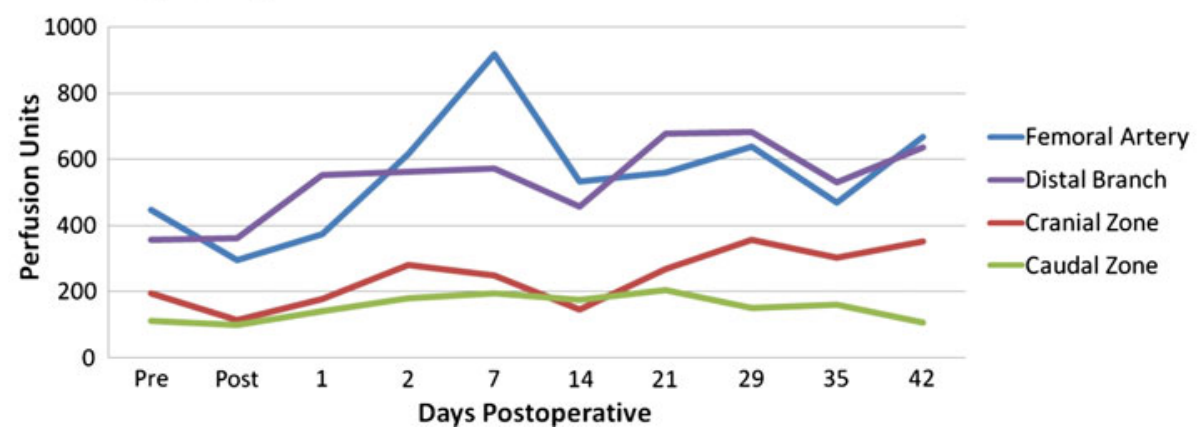




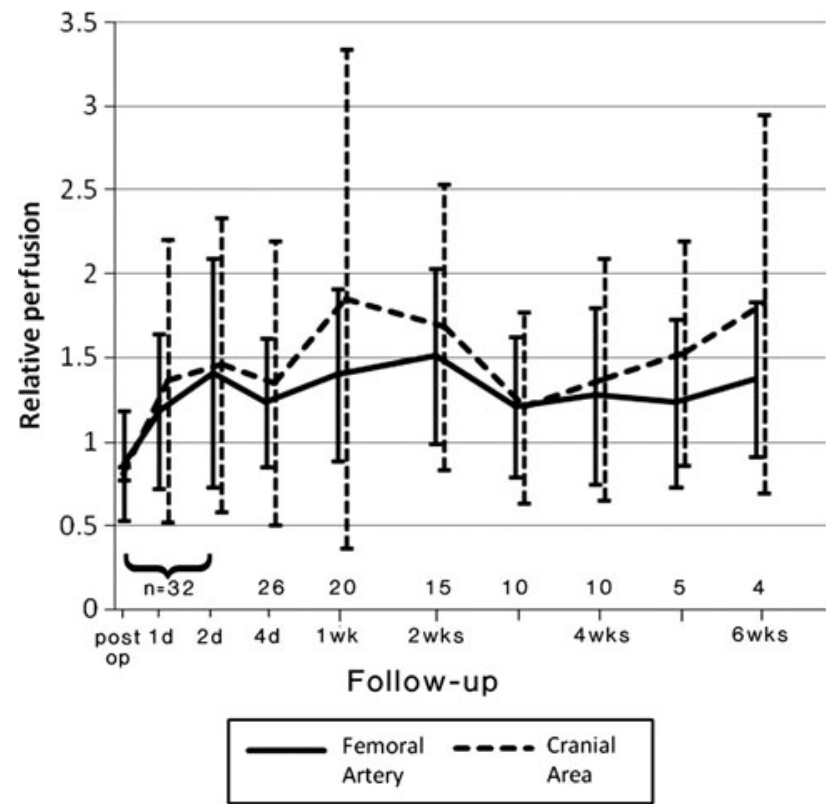

Fig. 5 Perfusion (relative to pre-operative measures) in femoral artery region and cranial region (adjacent to fracture); Pooled data for all animals and all time points

at day one was highly significantly different from postfracture perfusion, but not from the pre-fracture measure $(p=0.109)$.

Micro-CT scans indicated no clear changes in spatial distribution of the vessels over time of healing, with an even distribution of vessel densities throughout the region surveyed.

Fractured limbs at all time points displayed highly significantly greater vessel densities (vessel number) than their contralateral intact limbs $(p=0.005$, Student's paired $t$ test). The number of small vessels for each limb expressed as a fraction of the total number of vessels also showed a significantly greater proportion of smaller vessels on the fractured side (Fig. $6 ; p=0.021$, Student's paired $t$ test). This proportion also showed an increase from 2 days post-operatively to 14 days post-operatively, and then, the difference from the intact side diminished.

\section{Discussion}

This study is the first to use two-dimensional laser Doppler scanning longitudinally throughout the healing period, to show an immediate post-operative decrease in perfusion adjacent to the fracture site, with a subsequent increase, especially throughout the first 2 weeks of healing. By combining this with micro-CT scanning, we have shown that this was achieved by an increase in the number and proportion of small vessels adjacent to the fracture zone.

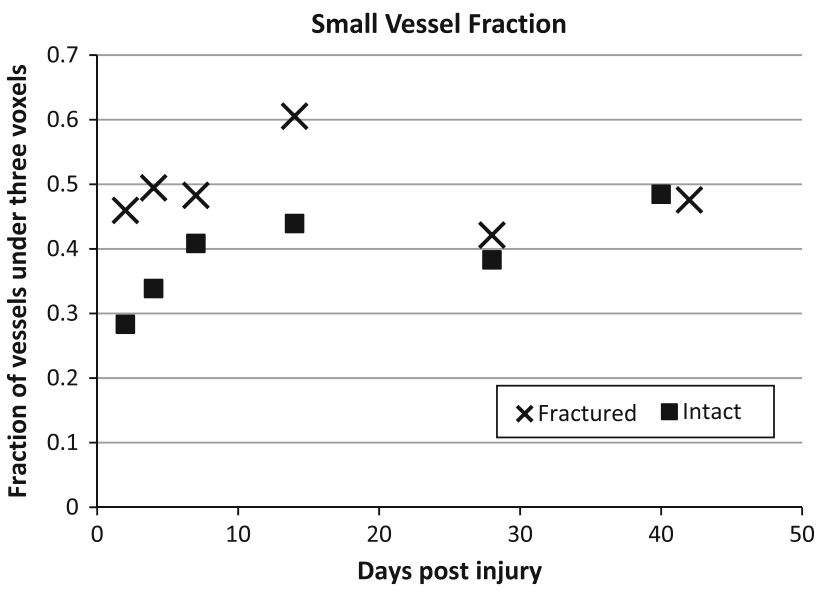

Fig. 6 Small blood vessel fraction (number of vessels $<3$ voxels/total number of vessels) in the fractured limb and contralateral intact limb) $(p=0.021$, Student's paired $t$ test $)$

This elaborates the findings of Matsumoto et al. [25] who used laser Doppler scanning of the whole hind limbs of mice to identify perfusion changes (post-fracture and 2 weeks later) and histology to measure vessel density. Others have used single-point measures by laser Doppler probe to impute perfusion in whole regions, also reporting increased perfusion after fracture [32, 50].

Our laser Doppler measurements confirm that neovascularisation begins soon (2-14 days) after fracture, particularly in the soft tissues surrounding the fracture (cranial region of interest in laser Doppler scans). This indicates that neovascularisation of the tissue occurs in a spatially targeted region, not just through general limb perfusion, and is temporally organised to provide quick supply of nutrients and blood to the fracture site. The micro-CT vessel analyses indicate that this increased perfusion is achieved by more and finer vessels and not just by vasodilation of existing vessels. The vessel distributions around the fracture site (along the axis of the femur) were predominantly even and showed no consistent variation or trend towards one side of the fracture line or location. Although the pins were placed in the blunt dissected surgical approach, it is not possible to exclude any vascular response to this or the cortical fixation of the pins.

Similar time trends were reported by Melnyk et al. [27], who also found increased perfusion shortly after fracture followed by a decline, in their study of perfusion in fracture healing with soft tissue damage, using a probe-based transcutaneous laser Doppler flowmeter measuring single points at the fracture site and one centimetre distally and proximally. Perfusion near the fracture site was only clearly greater than preoperatively at 3 and 7 days, and 14 days slightly greater. Perfusion at the fracture gap lagged behind-greater perfusion occurred at 7 and 14 days after fracture. 
Previous studies have shown similar trends in neovascularisation of soft tissue around the fracture callus, dating back to Gothman's studies in the 1960s [9-12]. These studies established clinical practice for treatment of displaced fractures by underlining the benefit of soft tissue neovascularisation. However, neovascularisation analyses were limited to ex vivo assessment of $2 \mathrm{D}$ angiographs or histology, or single-point measures of perfusion. By combining laser Doppler scanning and micro-CT analysis, we were able to provide $3 \mathrm{D}$ structural and $2 \mathrm{D}$ functional measurements of vascularisation, showing that neovascularisation occurred by angiogenesis (new vessel formation) and not only vasculogenesis (vasodilation of existing vessels). Micro-CT scanning undecalcified bones enabled localisation of the vascularity with respect to the fracture gap, although segmentation and scan analysis were thereby made more difficult. Our images indicate that angiogenesis (as evident by number of small vessels) was evenly distributed along the fractured bone length.

Our micro-CT results correlate with the findings of Tomlinson et al. [41], who also used Microfil infiltration and micro-CT to identify vessels in osteogenic response to overloading. Using anti-angiogenic treatment, they showed that angiogenesis was significant in the increased vascularity at 3 and 7 days after loading-induced stress fracturerelated osteogenesis. The use of Microfil to image vessels down to 10 microns was reported by Marxen et al. [24] and demonstrated by Vasquez et al. [44], working with similar 20 micron micro-CT resolution.

Neovascularisation of the soft tissues is critical as it provides a blood source for the healing fracture callus. In our current study, we were not able to visualise or quantify vessels directly within the fracture callus. The resolution and depth of penetration of the laser Doppler scan were insufficient to quantify the angiogenic processes in the callus. Though our micro-CT images most probably captured the intra-cortical vessels, the contrast between bone and vessels was insufficient to allow thresholding differentiation and segmentation. Further work will use histology and decalcified bones to examine the vessel structure in the bone and fracture callus.

Though variations in perfusion were large between animals (large standard deviations in Fig. 5), the temporal and spatial patterns of revascularisation were similar for all animals (statistical analysis, $p<0.05$ ). Large variation in blood perfusion measurements is not uncommon due to the numerous internal and external factors that influence perfusion [5]. Sample size at each time point was small $(n=5)$ but comparable to similar studies [50], and larger sample sizes would probably not change the overall trends seen.

The depth of penetration for laser Doppler scanning depends on the tissue type. Our preliminary data indicated that laser Doppler scanning can detect flow through $5 \mathrm{~mm}$ of muscle tissue, and Melnyk et al. [27] report laser penetration of bone to $2 \mathrm{~mm}$ depth and skin/muscle to $6 \mathrm{~mm}$. Perfusion images are weighted projections of perfusion as a function of depth (i.e., deeper vessels appear to have less flow). A few animals suffered skin nicks during shaving, which resulted in extremely high perfusion values despite relatively small skin injury. The injury caused by surgery was all on the lateral side of the leg, and scanning was performed on the medial side, minimising the skin effects of the surgical site.

Reed has shown that revascularisation at early time points is critical for ensuring healing [36]. In that rat model, delayed revascularisation resulted in atrophic nonunion. Now that we have developed methods for functional and structural characterisation of neovascularisation, future work will examine methods for increasing vascularity of the soft tissue in these early time points.

Laser Doppler scanning is a non-invasive in vivo measurement causing minimal distress to the animal which can be used for longitudinal studies of treatment effects and responses. Micro-CT complements the functional measures provided with laser Doppler with 3D structural information of the vessel network. Further investigation using these modalities in fracture healing studies may enable correlation with other factors that promote the healing process.

Acknowledgments The study was supported by a grant from the Medical Research Council.

Conflict of interest None.

Open Access This article is distributed under the terms of the Creative Commons Attribution License which permits any use, distribution, and reproduction in any medium, provided the original author(s) and the source are credited.

\section{References}

1. Abbotts R, Harrison SE, Cooper GL (2007) Primary blast injuries to the eye: a review of the evidence. J R Army Med Corps 153(2):119-123

2. Blanch RJ, Scott RA (2008) Primary blast injury of the eye. J R Army Med Corps 154(1):76

3. Brighton CT, Hunt RM (1991) Early histological and ultrastructural changes in medullary fracture callus. J Bone Jt Surg Am 73(6):832-847

4. Brighton CT, Hunt RM (1997) Early histologic and ultrastructural changes in microvessels of periosteal callus. J Orthop Trauma 11(4):244-253

5. Bumann M, Henke T, Gerngross H, Claes L, Augat P (2003) Influence of haemorrhagic shock on fracture healing. Langenbecks Arch Surg 388(5):331-338

6. ElMaraghy AW, Schemitsch EH, Richards RR (1998) Femoral and cruciate blood flow after retrograde femoral reaming: a canine study using laser Doppler flowmetry. J Orthop Trauma 12(4):253-258 
7. Garcia-Sanz A, Rodriguez-Barbero A, Bentley MD, Ritman EL, Romero JC (1998) Three-dimensional micro computed tomography of renal vasculature in rats. Hypertension 31(1 Pt 2):440-444

8. Gerber HP, Ferrara N (2000) Angiogenesis and bone growth. Trends Cardiovasc Med 10(5):223-228

9. Gothman L (1962) Local arterial changes associated with diastasis in experimental fractures of the rabbit's tibia treated with intramedullary nailing. A microangiographic study. Acta Chir Scand 123:104-110

10. Gothman L (1962) Local arterial changes caused by surgical exposure and the application of encircling wires (cerclage) on the rabbit's tibia. A microangiographic study. Acta Chir Scand 123:9-16

11. Grundnes O, Utvag SE, Reikeras O (1994) Restoration of bone flow following fracture and reaming in rat femora. Acta Orthop Scand 65(2):185-190

12. Hackenbroich $\mathrm{C}$, Wagner S, Windhagen H, Meyer-Lindenberg A (2008) Continual measurement of intramedullary blood perfusion with laser Doppler flowmetry in intact and ostectomized tibiae of rabbits. Lab Anim 42(3):305-319

13. Harry LE, Sandison A, Paleolog EM, Hansen U, Pearse MF, Nanchahal J (2008) Comparison of the healing of open tibial fractures covered with either muscle or fasciocutaneous tissue in a murine model. J Orthop Res 26(9):1238-1244

14. Hausman MR, Schaffler MB, Majeska RJ (2001) Prevention of fracture healing in rats by an inhibitor of angiogenesis. Bone 29(6):560-564

15. Hinsley DE, Hobbs CM, Watkins PE (2002) The role of laser Doppler flowmetry in assessing the viability of bone fragments in an open fracture. Injury 33(5):435-438

16. Hupel TM, Aksenov SA, Schemitsch EH (1998) Effect of limited and standard reaming on cortical bone blood flow and early strength of union following segmental fracture. J Orthop Trauma 12(6):400-406

17. Jain R, Podworny N, Hearn T, Anderson GI, Schemitsch EH (1997) Effect of stainless steel and titanium low-contact dynamic compression plate application on the vascularity and mechanical properties of cortical bone after fracture. J Orthop Trauma 11(7):490-495

18. Kan Z, Kobayashi S, Phongkitkarun S, Charnsangavej C (2005) Functional CT quantification of tumour perfusion after transhepatic arterial embolization in a rat model. Radiology 237(1):144-150

19. Kan Z, Phongkitkarun S, Kobayashi S, Tang Y, Ellis LM, Lee TY, Charnsangavej C (2005) Functional CT for quantifying tumour perfusion in antiangiogenic therapy in a rat model. Radiology 237(1):151-158

20. Kindlmann GL, Weinstein DM, Jones GM, Johnson CR, Capecchi MR, Keller C (2005) Practical vessel imaging by computed tomography in live transgenic mouse models for human tumours. Mol Imaging 4(4):417-424

21. Komuta K, Hirano T, Iwasaki K (1998) Structural changes in blood vessels entering the growth plate during growth in rats. Int Orthop 22(1):11-18

22. Lienau J, Schell H, Duda GN, Seebeck P, Muchow S, Bail HJ (2005) Initial vascularization and tissue differentiation are influenced by fixation stability. J Orthop Res 23(3):639-645

23. Mark H, Penington A, Nannmark U, Morrison W, Messina A (2004) Microvascular invasion during endochondral ossification in experimental fractures in rats. Bone 35(2):535-542

24. Marxen M, Thornton MM, Chiarot CB, Klement G, Koprivnikar J, Sled JG, Henkelman RM (2004) Micro-CT scanner performance and considerations for vascular specimen imaging. Med Phys 31(2):305-313

25. Matsumoto T, Mifune Y, Kawamoto A, Kuroda R, Shoji T, Iwasaki H, Suzuki T, Oyamada A, Horii M, Yokoyama A,
Nishimura H, Lee SY, Miwa M, Doita M, Kurosaka M, Asahara $\mathrm{T}$ (2008) Fracture induced mobilization and incorporation of bone marrow-derived endothelial progenitor cells for bone healing. J Cell Physiol 215(1):234-242

26. Matsuyama J, Ohnishi I, Kageyama T, Oshida H, Suwabe T, Nakamura K (2005) Osteogenesis and angiogenesis in regenerating bone during transverse distraction: quantitative evaluation using a canine model. Clin Orthop Relat Res 433:243-250

27. Melnyk M, Henke T, Claes L, Augat P (2008) Revascularisation during fracture healing with soft tissue injury. Arch Orthop Trauma Surg 128(10):1159-1165

28. Mesa RA, Verstovsek S, Cervantes F, Barosi G, Reilly JT, Dupriez B, Levine R, Le Bousse-Kerdiles MC, Wadleigh M, Campbell PJ, Silver RT, Vannucchi AM, Deeg HJ, Gisslinger H, Thomas D, Odenike O, Solberg LA, Gotlib J, Hexner E, Nimer SD, Kantarjian H, Orazi A, Vardiman JW, Thiele J, Tefferi A (2007) Primary myelofibrosis (PMF), post polycythemia vera myelofibrosis (postPV MF), post essential thrombocythemia myelofibrosis (post-ET $\mathrm{MF})$, blast phase PMF (PMF-BP): consensus on terminology by the international working group for myelofibrosis research and treatment (IWG-MRT). Leuk Res 31(6):737-740

29. Mohanti RC, Mahakul NC (1983) Vascular response in fractured limbs with and without immobilisation: an experimental study on rabbits. Int Orthop 7(3):173-177

30. Moore DF, Jerusalem A, Nyein M, Noels L, Jaffee MS, Radovitzky RA (2009) Computational biology-modelling of primary blast effects on the central nervous system. Neuroimage 47(Suppl 2): T10-T20

31. Mu HJ, Xie R, Shen YF, Jiang YQ, Zeng YJ (2009) Cadherin-13 in primary and blast crisis chronic myeloid leukaemia: declining expression and negative correlation with the BCR/ABL fusion gene. Br J Biomed Sci 66(1):20-24

32. Murnaghan M, Li G, Marsh DR (2006) Nonsteroidal antiinflammatory drug-induced fracture nonunion: an inhibition of angiogenesis? J Bone Jt Surg Am 88(Suppl 3):140-147

33. Prior BM, Lloyd PG, Ren J, Li H, Yang HT, Laughlin MH, Terjung RL (2004) Time course of changes in collateral blood flow and isolated vessel size and gene expression after femoral artery occlusion in rats. Am J Physiol Heart Circ Physiol 287(6):H2434-H2447

34. Raina AK, Baheti M, Haldar A, Ramulu M, Chakraborty AK, Sahu PB, Bandopadhayay C (2004) Impact of blast induced transitory vibration and air-overpressure/noise on human brainan experimental study. Int J Environ Health Res 14(2):143-149

35. Rawool NM, Goldberg BB, Forsberg F, Winder AA, Hume E (2003) Power Doppler assessment of vascular changes during fracture treatment with low-intensity ultrasound. J Ultrasound Med 22(2):145-153

36. Reed AA, Joyner CJ, Isefuku S, Brownlow HC, Simpson AH (2003) Vascularity in a new model of atrophic nonunion. J Bone Jt Surg Br 85(4):604-610

37. Rhinelander FW (1974) Tibial blood supply in relation to fracture healing. Clin Orthop Relat Res 105:34-81

38. Ritenour AE, Baskin TW (2008) Primary blast injury: update on diagnosis and treatment. Crit Care Med 36(7 Suppl):S311-S317

39. Seo Y, Hashimoto T, Nuki Y, Hasegawa BH (2008) In vivo micro-CT imaging of rodent cerebral vasculature. Phys Med Biol 53(7):N99-N107

40. Tomlinson RE, McKenzie JA, Schmieder AH, Wohl GR, Lanza GM, Silva MJ (2013) Angiogenesis is required for stress fracture healing in rats. Bone 52(1):212-219

41. Tomlinson RE, McKenzie JA, Schmieder AH, Wohl GR, Lanza GM, Silva MJ (2013) Angiogenesis is required for stress fracture healing in rats. Bone 52(1):212-219

42. Trueta $\mathbf{J}(1963)$ The role of the vessels in osteogenesis. J Bone Jt Surg 45-B(2):402-418 
43. Trueta J (1974) Blood supply and the rate of healing of tibial fractures. Clin Orthop Relat Res 105:11-26

44. Vasquez SX, Gao F, Su F, Grijalva V, Pope J, Martin B, Stinstra J, Masner M, Shah N, Weinstein DM, Farias-Eisner R, Reddy ST (2011) Optimization of micro-CT imaging and blood vessel diameter quantitation of preclinical specimen vasculature with radiopaque polymer injection medium. PLoS One 6(4)

45. Wallace AL, Draper ER, Strachan RK, McCarthy ID, Hughes SP (1991) The effect of devascularisation upon early bone healing in dynamic external fixation. J Bone Jt Surg Br 73(5):819-825

46. Warden DL, French LM, Shupenko L, Fargus J, Riedy G, Erickson ME, Jaffee MS, Moore DF (2009) Case report of a soldier with primary blast brain injury. Neuroimage 47(Suppl 2): T152-T153
47. Winet H (1996) The role of microvasculature in normal and perturbed bone healing as revealed by intravital microscopy. Bone 19(1 Suppl):39S-57S

48. Yang HT, Deschenes MR, Ogilvie RW, Terjung RL (1996) Basic fibroblast growth factor increases collateral blood flow in rats with femoral arterial ligation. Circ Res 79(1):62-69

49. Zhang L, Bail H, Mittlmeier T, Haas NP, Schaser KD (2003) Immediate microcirculatory derangements in skeletal muscle and periosteum after closed tibial fracture. J Trauma 54(5):979-985

50. Zheng LW, Ma L, Cheung LK (2008) Changes in blood perfusion and bone healing induced by nicotine during distraction osteogenesis. Bone 43(2):355-361 